\title{
A comparison of methods to determine bilateral asymmetries in vertical leg stiffness
}

Sean J. Maloney, Iain M. Fletcher, Joanna Richards

Department of Sports Science and Physical Activity, University of Bedfordshire, Bedford, United Kingdom

Corresponding author

Sean Maloney, Research Graduate School, University of Bedfordshire, Polhill Avenue, Bedford, MK41 9EA

Sean.Maloney@beds.ac.uk

$+447800810099$

Keywords

leg-spring behaviour, centre of mass displacement, spring-mass model

\section{Acknowledgements}

No benefits in any form have been or will be received from any commercial party or grant body related directly or indirectly in relation to this manuscript. The results of the current study do not constitute the endorsement of any product by the authors. 


\section{Abstract}

Whilst the measurement and quantification of vertical leg stiffness (Kvert) asymmetry is of important practical relevance to athletic performance, literature investigating bilateral asymmetry in $\mathrm{K}_{\text {vert }}$ is limited. Moreover, how the type of task used to assess $K_{\text {vert }}$ may affect the expression of asymmetry has not been properly determined. Twelve healthy males performed three types of performance task on a dual force plate system to determine $\mathrm{K}_{\text {vert }}$ asymmetries; the tasks were: a) bilateral hopping, b) bilateral drop jumping, and c) unilateral drop jumping. Across all three methods, Kvert was significantly different between compliant and stiff limbs $(P<0.001)$ with a significant interaction effect between limb and method $(P=0.005)$. Differences in Kvert between compliant and stiff limbs were $-5.3 \%(P<0.001),-21.8 \%(P=0.007)$ and $-15.1 \%(P<0.001)$ for the bilateral hopping, bilateral drop jumping and unilateral drop jumping methods respectively. All three methods were able to detect significant differences between compliant and stiff limbs, and could be used as a diagnostic tool to assess $\mathrm{K}_{\text {vert }}$ asymmetry. Drop jumping tasks detected larger Kvert asymmetries than hopping, suggesting that asymmetries may be expressed to a greater extent in acyclic, maximal performance tasks. 


\section{Introduction}

Stiffness describes the resistance of an object to deformation (Brughelli \& Cronin, 2008). Specifically, vertical leg stiffness (Kvert) may be described by changes in the body's centre of mass in response to force (Pearson \& McMahon, 2012). Although the role of $\mathrm{K}_{\text {vert }}$ in modulating injury risk and athletic performance may be well established (Butler, Crowell III, \& Davis, 2003; Pearson \& McMahon, 2012), literature investigating bilateral asymmetry in leg stiffness is limited. A significant link between Kvert asymmetry and soft-tissue injury has been reported by Pruyn et al. (2012) and such asymmetry may also be expected to impair athletic performance (Wilson, Murphy, \& Pryor, 1994). Whilst it is important to note that the latter hypothesis has not been properly explored, it is clear that the measurement and quantification of $\mathrm{K}_{\text {vert }}$ asymmetry is of important practical relevance to athletic performance.

Kvert may be assessed during a variety of performance tasks, including running (Coleman, Cannavan, Horne, \& Blazevich, 2012) and drop jumping (Arampatzis, Schade, Walsh, \& Brüggemann, 2001), but is most commonly assessed during the performance of a bilateral 'hopping' task (Hobara, Inoue, Kobayashi, \& Ogata, 2014; Joseph, Bradshaw, Kemp, \& Clark, 2013). During hopping tasks, individuals are required to perform an uninterrupted sequence of repeated bilateral jumps on a force plate. Measurements of vertical ground reaction force (vGRF) and negative displacement of the centre of mass (COM) are recorded and $\mathrm{K}_{\text {vert }}$ is subsequently calculated (Hobara et al., 2014; Joseph et al., 2013). Hopping tasks have been shown to differentiate between certain groups, for example, it has been demonstrated that power-trained athletes exhibit greater $K_{v e r t}$ than endurance-trained athletes (Hobara et al., 2008) and that endurance-trained athletes exhibit greater Kvert than untrained individuals (Hobara et al., 2010). Pruyn, Watsford, and Murphy 
(2014) split a cohort of female team-sport athletes into high Kvert and low Kvert groups; whilst inter-group differences were not significant, performances in a number of speed and power tests were superior in the high $\mathrm{K}_{\text {vert }}$ group and were reported with large effect sizes $(d>0.7)$.

One potential issue with hopping tasks is that they are typically performed at set hopping frequencies and are inherently submaximal in nature (Hobara et al., 2014; Joseph et al., 2013). As such, bilateral hopping tasks may demonstrate greater correspondence to sub-maximal cyclic performances, such as endurance running, rather than short-term maximal performances, such as jumping. For this reason, it may be desirable to assess $\mathrm{K}_{\text {vert }}$ during a maximal performance task such as a drop jump. Given that the drop jump is a acyclic action performed with the intent to maximise jump height whilst minimising ground contact time (Marshall \& Moran, 2013), it may carry greater ecologically validity as an assessment tool for leg stiffness when compared to hopping tasks and be more representative of single maximal jumping effort (Flanagan \& Harrison, 2007). Whilst Kvert has been modelled during drop jumping by Arampatzis et al. (2001), this task has not been used to examine relationships with performance or to examine inter-group differences in the same way as bilateral hopping tasks. Further research is required to determine if Kvert values achieved during drop jumping demonstrate similar relationships with performance and training status as those achieved during bilateral hopping.

As previously highlighted, literature investigating bilateral asymmetry in $\mathrm{K}_{\text {vert }}$ is limited. Bachman, Heise, and Bressel (1999), Heise and Bachman (2000) and Divert, Baur, Mornieux, Mayer, and Belli (2005) all observed no significant Kvert asymmetries during running, although the cyclic, submaximal limb action and bilateral nature of locomotion may be expected to encourage symmetry. When the 
results of Bachman et al. (1999) are presented as a symmetry angle, a method used to quantify asymmetry (Zifchock, Davis, Higginson, \& Royer, 2008), average differences between the left and right limbs were $-3.8 \%$ and $-2.7 \%$ at running speeds of $3.5 \mathrm{~m} . \mathrm{s}^{-1}$ and $5.3 \mathrm{~m} . \mathrm{s}^{-1}$, respectively. Similarly, Hobara, Inoue, and Kanosue (2013) did not report significant Kvert asymmetries between non-dominant and dominant limbs during unilateral hopping; average differences of $-4.9 \%, 1.1 \%$ and $3.0 \%$ were observed at hopping frequencies of $1.5 \mathrm{~Hz}, 2.2 \mathrm{~Hz}$ and $3 \mathrm{~Hz}$, respectively.

Flanagan and Harrison (2007) compared asymmetries during unilateral drop jumps and repeated drop jumps performed on a sledge apparatus. The investigators reported that no asymmetries were apparent during the cyclic, repeated jumps, however, significant asymmetry in reactive strength index - closely linked to leg stiffness (Flanagan \& Comyns, 2008) - was evident during the acyclic drop jump task. When presented as a symmetry angle, average differences in Kvert between limbs were $-1.1 \%$ for drop jumping and $0.4 \%$ for repeated drop jumping. Whilst the observations of Flanagan and Harrison (2007) demonstrate that the type of performance task chosen to assess stiffness carries the potential to modulate how asymmetries may be expressed, further research is necessary to elucidate this effect.

As cyclic, submaximal versus acyclic, maximal performance tasks may differently express asymmetries, so too may bilateral versus unilateral performance tasks. Benjanuvatra, Lay, Alderson, and Blanksby (2013) compared impulses of the left and right limbs during bilateral and unilateral countermovement jumping, finding that asymmetries presented in the bilateral jump did not correspond to asymmetries in the unilateral jump. For example, individuals may express a right-side dominance 
during the bilateral task but a left-side dominance in the unilateral task. These observations led the investigators to conclude that asymmetry in bilateral tasks is driven by neural factors, a proposition supported by earlier investigations conducted by Simon and Ferris (2008). As unilateral jumping tasks rely on the extension forces generated from a single limb such tasks would appear to be a more suitable choice if seeking to quantify functional parameters of the limb such as Kvert. However, such propositions are yet to have been evaluated by the literature and further research is required to explore this hypothesis.

The purpose of the current study was to investigate the prevalence of bilateral asymmetry in $K_{\text {vert }}$ during three different performance tasks: a) bilateral hopping, b) bilateral drop jumping, and c) unilateral drop jumping. It was hypothesised that asymmetries would be significantly greater in the maximal drop jump versus the submaximal hopping task and that asymmetries would be significantly greater in the unilateral versus bilateral drop jump. 


\section{Method}

\section{Participants}

Twelve healthy males (age: $22 \pm 2$ years; height: $1.77 \pm 0.06 \mathrm{~m}$; body mass: $74.0 \pm$ $7.9 \mathrm{~kg}$ ) volunteered to participate in the study. Participants were recreationally active (undertaking $\geq 2.5$ hours of physical activity per week), reported no previous (within the last 12 months) or present lower limb injury and provided informed consent to participate in the study. Full ethical approval was granted by [REMAINDER OF SENTENCE REMOVED FOR ANONYMOUS PEER REVIEW PURPOSE]. All procedures were conducted in accordance with the Declaration of Helsinki.

\section{Experimental trials}

A familiarisation session was performed seven days prior to the experimental trial; pilot studies within the same experimental cohort had indicated that a single familiarisation session was appropriate for all testing methods and experimental variables. The familiarisation session was a complete simulation of the experimental trial outlined below.

All trials were conducted at the same time of day (09:30 - 11:00) for each participant, to alleviate the effects of circadian rhythms. The testing laboratory was controlled at an ambient temperature of $25^{\circ} \mathrm{C}$. Participants were instructed to prepare for testing as they would for training; nutrition, hydration and sleep were not monitored. The execution of each experimental trial was monitored by a United Kingdom Strength and Conditioning Association accredited strength and conditioning coach to ensure for consistency of technique. 


\section{Warm-up}

All participants completed the same warm-up procedure outlined in Table I. The warm-up procedure consisted of 15 dynamic exercises progressing from low to high intensities and from generic to specific movement patterns.

\section{*** Table 1 Here ${ }^{* * *}$}

A rest period of 60 seconds was prescribed between each of the exercises from the specific movement preparation phase of the warm-up; all other exercises were not prescribed with rest periods. A rest period of 180 seconds was prescribed between the termination of the warm-up and commencement of the testing protocol.

\section{Testing}

All $K_{\text {vert }}$ assessments were performed on a duel force plate system (Kistler 9281, Kistler Instruments, Winterthur, Switzerland) with data recorded independently for the left and right limbs. The plates each measured $0.6 \mathrm{~m} \times 0.4 \mathrm{~m}$, were set flush into the laboratory floor as per manufacturer guidelines and spaced by a distance of 0.1 m. Kinetic data was sampled at $1000 \mathrm{~Hz}$ and saved with the use of the manufacturer supplied software (BioWare 3.24, Kistler, Winterthur, Switzerland) for later analysis. The vGRF traces were filtered with a low-pass Butterworth filter (cut-off frequency: $100 \mathrm{~Hz}$ ) using the BioWare software.

\section{Bilateral hopping testing}

Unshod participants performed a series of 30 consecutive bilateral hops. Participants performed two hopping trials in each experimental trial; these were separated by a recovery period of 180 seconds. Hops were performed at a self-selected frequency (mean hopping frequency: $2.8 \pm 0.3 \mathrm{~Hz}$; mean ground contact time: $0.175 \pm 0.023$ 
sec) as pilot testing indicated that participants were unable to satisfactorily perform the task at a set hopping frequency of $2.2 \mathrm{~Hz}$. Participants were instructed to "hop on the balls of your feet at a constant rhythm." Only bilateral hopping trials were performed as pilot testing indicated that participants were unable to maintain a rhythmic frequency when hopping unilaterally. Five consecutive hops from $6^{\text {th }}$ to the $10^{\text {th }}$ hop were sampled for data collection (Hobara et al., 2014). The ground contact time of each of the 5 hops was required to fall within $\pm 5 \%$ of the average ground contact time for the 5 hop sample (Moresi, Bradshaw, Greene, \& Naughton, 2015); all hopping trials met this criteria.

\section{Drop jump testing}

Following a recovery period of 180 seconds, participants performed three unshod bilateral drop jumps and three unshod unilateral drop jumps for each limb. The order in which participants performed bilateral and unilateral drop jumps was counterbalanced. For the execution of all drop jumps, participants stepped off a 0.18 $\mathrm{m}$ box and performed a vertical jump immediately upon landing. Participants were instructed to: a) step, not jump, off the box, and b) minimise ground contact time during the landing phase; the execution of every jump was monitored for consistency of technique. The box height of $0.18 \mathrm{~m}$ was chosen as participants were unable to minimise ground contact time effectively at additional height increments $(0.30 \mathrm{~m}$ and $0.45 \mathrm{~m}$ ) during pilot testing. For the bilateral drop jump, participants stepped off the box with their preferred foot; this foot was established in the participants' familiarisation trial and remained consistent thereafter. For the unilateral drop jump, participants stepped off the box with the designated foot for that trial. Each repetition of the drop jump was separated by 60 seconds to facilitate recovery between efforts (Read \& Cisar, 2001). 


\section{Data analysis}

The instants of touchdown and take-off during the hopping and drop jump trials were determined based on a $10 \mathrm{~N}$ vGRF threshold (Lloyd, Oliver, Hughes, \& Williams, 2009). Inverse dynamics was used to express acceleration, velocity and negative displacement of the COM; this was determined from the vertical force trace using the equations described by Blazevich (2007). Kvert was calculated as the ratio of peak vGRF relative to the peak negative displacement of the COM during the initial ground contact phase (Farley \& Morgenroth, 1999); this was averaged over the five sampled hops or the three recorded drop jumps. In an effort to ensure the efficacy of the spring-mass model, the force-displacement correlation coefficient of each trial was required to be $\geq 0.8$ (Padua, Arnold, Carcia, \& Granata, 2005); all trials met this criteria. As $\mathrm{K}_{\text {vert }}$ is affected by body size, $\mathrm{K}_{\text {vert }}$ values were reported relative to body mass (Farley, Glasheen, \& McMahon, 1993).

Between-session coefficients of variation for the three variables and three methods were established using the same experimental cohort; the values for bilateral hopping, bilateral drop jumping and unilateral drop jumping, respectively, were $3 \%$, $5 \%$ and $2 \%$ for vGRF, $12 \%, 12 \%$ and $6 \%$ for negative COM displacement and $14 \%$, $13 \%$ and $8 \%$ for Kvert.

\section{Statistical analysis}

Limbs were independently categorised as either stiff or compliant based upon the Kvert values achieved within each of the three testing methods. Asymmetries were quantified using the symmetry angle ( $\left.\theta_{\text {SYM }}\right)$, calculated using the procedures outlined by Zifchock et al. (2008). The ${ }^{\theta_{\text {SYM }}}$ is able to identify inter-limb differences in a similar manner to other asymmetry indices, such as the symmetry index (Robinson, Herzog, 
\& Nigg, 1987), but reduces the likelihood of artificially inflated values, treats positive and negative values as equal and opposite in magnitude, does not require a reference value and provides a standard scale for interpretation (Zifchock et al., 2008). As $\theta_{S Y M}$ values may be negative or positive to reflect left or right side dominance, negative values were transformed to positive values prior to statistical analysis in order to evaluate differences solely in the magnitude of asymmetry.

Shapiro-Wilks tests were performed to assess for normality; all variables were considered to be normally distributed given an alpha level of $P>0.05$. A $2 \times 3$ (limb versus method) repeated-measures analysis of variance (ANOVA) was used to test for differences between methods, the effect size measured using Eta-squared $\left(\eta^{2}\right)$, and Sidak post-hoc analyses performed where appropriate. A 1 × 3 ANOVA was performed to analyse for differences in $\theta_{\text {SYM }}$ between methods. Pair-wise effect sizes (d) (Cohen, 1998) were also calculated and interpreted using the thresholds defined by Rhea $(2004)$ (trivial $=<0.25 ;$ small $=0.25-0.50 ;$ moderate $=0.50-1.0 ;$ large $=>$ 1.0). All ANOVAs were conducted using the Statistical Package for the Social Sciences for Windows (v19.0; SPSS Inc., Chicago, USA) with an alpha level of $P \leq$ 0.05 


\section{Results}

\section{${ }^{* * *}$ Table 2 Here ${ }^{* * *}$}

Vertical ground reaction force

Landing vGRF was significantly different between methods $\left(F_{2,22}=63.07 ; \eta^{2}=.851\right.$; $P<.001$ ) (Table 2). Landing vGRF was lower in bilateral hopping than in bilateral drop jumping $(-15.7 \% ; d=2.40 ; P=.005)$ and unilateral drop jumping $(-38.4 \% ; d=$ 3.44; $P<.001)$. Landing vGRF was lower in bilateral drop jumping than in unilateral drop jumping $(-27.0 \% ; d=1.04 ; P<.001)$.

Landing vGRF values were significantly different between the compliant and stiff $\operatorname{limbs}\left(F_{1,11}=6.83 ; \eta^{2}=.383 ; P=.024\right)$, there was no significant interaction effect between $\operatorname{limb}$ and method $\left(\mathrm{F}_{2,22}=1.91 ; \eta^{2}=.148 ; P=.172\right)$. In bilateral hopping, landing vGRF was $2.9 \%$ lower in the compliant limb versus the stiff limb $(d=0.23 ; P$ $=.002)$. In bilateral drop jumping and unilateral drop jumping, differences between limbs were not significant (bilateral drop jumping: $9.7 \% ; d=0.57 ; P=.071$ and unilateral drop jumping: $2.4 \% ; d=0.16 ; P=.163)$.

vGRF $\theta_{\text {SYM }}$ was significantly different between methods $\left(F_{2,22}=4.37 ; \eta^{2}=0.28 ; P=\right.$ .025). There were no significant pair-wise differences in vGRF $\theta_{\text {SYM }}$ between bilateral hopping and bilateral drop jumping $(d=1.50 ; P=.091)$, bilateral hopping and unilateral drop jumping $(d=0.25 ; \mathrm{P}=.378)$, and bilateral drop jumping and unilateral drop jumping $(d=1.24 ; P=.274)$.

Negative centre of mass displacement

Negative COM displacement was significantly different between methods $\left(F_{2,22}=\right.$ 69.86; $\left.\eta^{2}=.864 ; P<.001\right)$ (Table 2). Displacement was greater in bilateral hopping 
than in bilateral drop jumping $(71.7 \% ; d=4.42 ; P<.001)$ and in unilateral drop jumping $(79.2 \% ; d=4.75 ; P<.001)$. Differences between negative COM displacement in bilateral drop jumping and unilateral drop jumping were not significant $(26.7 \% ; d=0.34 ; P=.610)$.

Negative COM displacement was significantly different between compliant and stiff $\operatorname{limbs}\left(F_{1,11}=18.34 ; \eta^{2}=.625 ; P=.001\right)$, there was no significant interaction effect between limb and method $\left(F_{2,22}=0.59 ; \eta^{2}=.051 ; P=.56\right)$. Differences between compliant and stiff limbs were $3.9 \%(d=0.13 ; P=.002), 25.1 \%(d=0.48 ; P=.002)$ and $19.8 \%(d=0.62 ; P=.002)$ for the bilateral hopping, bilateral drop jumping and unilateral drop jumping methods respectively.

Negative COM displacement $\theta_{\text {SYM }}$ was significantly different between methods $\left(F_{2,22}\right.$ $\left.=4.68 ; \eta^{2}=0.30 ; P=.020\right)$. Negative COM displacement $\theta_{S Y M}$ was less in both bilateral drop jumping $(d=1.43 ; P=.022)$ and unilateral drop jumping $(d=0.80 ; P=$ .180) when compared to bilateral hopping, and less in unilateral drop jumping when compared to bilateral drop jumping $(d=0.63 ; P=.024)$.

\section{Vertical leg stiffness}

$K_{\text {vert }}$ was significantly different between methods $\left(F_{2,22}=33.46 ; \eta^{2}=.753 ; P<.001\right)$ (Table 2). Kvert was lower in bilateral hopping than in bilateral drop jumping (-85.3\%; $d=1.05 ; P=.001)$ and in unilateral drop jumping $(-89.3 \% ; d=3.21 ; P<.001)$. Differences in $\mathrm{K}_{\text {vert }}$ between bilateral drop jumping and unilateral drop jumping were not significant $(-27.0 \% ; d=2.16 ; P=.095)$.

Kvert was significantly different between compliant and stiff limbs $\left(F_{1,11}=27.85 ; \eta^{2}=\right.$ $.717 ; P<.001)$ with a significant interaction effect between limb and method $\left(F_{2,22}=\right.$ 
$\left.6.19 ; \eta^{2}=.386 ; P=.005\right)$. Differences between compliant and stiff limbs were $-5.3 \%$ (d: $0.20 ; P<0.001),-21.8 \%(d=0.42 ; P=.007)$ and $-15.1 \%(d=0.50 ; P<.001)$ for the bilateral hopping, bilateral drop jumping and unilateral drop jumping methods respectively.

*** Table 3 Here ***

$\mathrm{K}_{\text {vert }} \theta_{\text {SYM }}$ was significantly different between methods $\left(F_{2,22}=4.68 ; \eta^{2}=.30 ; P=.020\right)$ (Table 3$). K_{\text {vert }}{ }^{\theta_{\text {SYM }}}$ was greater in bilateral drop jumping than bilateral hopping $(d=$ 1.38; $P=.032$ ); there were no significant pair-wise differences between bilateral hopping and unilateral drop jumping $(d=0.85 ; P=.145)$, and bilateral drop jumping and unilateral drop jumping $(d=0.52 ; P=.705)$. 


\section{Discussion}

The purpose of the current study was to investigate the prevalence of bilateral asymmetry in $\mathrm{K}_{\text {vert }}$ during three different types of performance task. It was hypothesised that asymmetries would be significantly greater in the maximal drop jump versus the submaximal hopping task and that asymmetries would be significantly greater in the unilateral versus bilateral drop jump. This study reported that significant $\mathrm{K}_{\text {vert }}$ asymmetries were observed within all three tasks, however, asymmetries observed in the bilateral drop jumping task was significantly larger than in the bilateral hopping task $(d=1.38 ; P=0.032)$. This was the first study to examine how the type of performance task may affect the expression of $\mathrm{K}_{\text {vert }}$ asymmetry.

The current study reported that all three performance tasks were able to detect significant asymmetries in $\mathrm{K}_{\text {vert; }}$ as such, all three tasks could be used as a diagnostic tool to directly assess and quantify Kvert asymmetry. Given that forcedisplacement correlations for all three methods were greater than 0.8 , it may also be determined that they all represent the simple spring-mass model effectively (Padua et al., 2005). It was shown that the two acyclic, maximal performance tasks (bilateral and unilateral drop jumps) detected larger Kvert asymmetries than the cyclic, submaximal task (bilateral hopping), although this difference was not significant for the unilateral drop jump despite a moderate-large effect size $(d=0.85 ; P=0.145)$. Using the same experimental cohort, between-session coefficients of variation for $K_{\text {vert }}$ of $14 \%, 13 \%$ and $8 \%$ were established for bilateral hopping, bilateral drop jumping and unilateral drop jumping respectively. The results of the current study should be interpreted with this is mind, particularly given that individual participants' Kvert asymmetries during bilateral hopping rarely exceed 0.5 of the coefficient of variation. 
Flanagan and Harrison (2007) reported no asymmetry in Kvert to be evident during acyclic or cyclic single leg sledge jumps but did demonstrate an asymmetry in reactive strength index - a property closely linked to Kvert (Flanagan \& Comyns, 2008) - to be expressed during the acyclic jump. The findings of the current study, in addition to the observations of Flanagan and Harrison (2007), suggest that asymmetries are differently expressed during acyclic, maximal performance tasks and cyclic, submaximal performance tasks. Whilst it may appear that acyclic, maximal performance tasks are superior for identifying $\mathrm{K}_{\text {vert }}$ asymmetry within individual athletes, careful consideration should be given to how the limbs will be required to function during sporting performance. For example, cyclic, submaximal tests, such as bilateral hopping, would be expected to be more representative assessment of $\mathrm{K}_{\text {vert }}$ asymmetry in endurance runners given a greater correspondence of the test to the submaximal, cyclic action of locomotion. The potential impact of increasing drop jump intensity (i.e. increasing the height of the box and subsequent vGRF upon landing) was not examined in the current study due to the training/skill level of the participants and should be explored in future investigations. Whilst intuitively it may seem that increasing intensity would result in larger $\mathrm{K}_{\text {vert }}$ asymmetries, this relationship has not been observed during unilateral hopping (Hobara et al., 2013).

It is important to note that the limb identified as the stiff limb for an individual within each performance task was not always the same limb (Table 3). For example, an individual may demonstrate greater $\mathrm{K}_{\mathrm{vert}}$ in the right limb during the bilateral drop jump but greater $\mathrm{K}_{\text {vert }}$ in the left limb during the unilateral drop jump. Benjanuvatra et al. (2013) reported similar findings for vGRF impulse asymmetries during bilateral and unilateral countermovement jumping, hypothesising that asymmetries during the 
bilateral jump were governed by a neural control mechanism in agreement with previous conclusions drawn by Simon and Ferris (2008). Ultimately, unilateral jumping performance is reliant solely on the forces transferred and generated through a single limb as opposed to an inter-limb 'trade-off' that is apparent during bilateral jumping. Moreover, as the current study demonstrated that the unilateral drop jump elicited the greatest absolute values of VGRF and $\mathrm{K}_{\mathrm{vert}}$, it may be inferred that the unilateral drop jump imposes a greater mechanical load on the lower limb. For these reasons, the unilateral drop jump would appear to be a superior task for the assessment of asymmetries in maximal limb properties such as $\mathrm{K}_{\text {vert. }}$

Kvert is a direct function of VGRF and negative COM displacement during the ground contact phase of the hop or jump (Hobara et al., 2014; Joseph et al., 2013). Asymmetries in $\mathrm{K}_{\text {vert }}$ are therefore a consequence of asymmetries in vGRF and/or negative COM displacement. Whilst small but significant differences in vGRF $(2.9 \%$; $P=0.002)$ was observed between the stiff and compliant limbs during bilateral hopping and bilateral drop jumping $(9.7 \% ; P=0.07)$, no such asymmetry was detected during unilateral drop jumping. It is apparent that $\mathrm{K}_{\text {vert }}$ asymmetries observed during the bilateral performance tasks were partially dependant on vGRF asymmetries, whereas this was not the case during the unilateral performance task.

Significant between-limb differences for negative COM displacement were observed during all three performance tasks. For bilateral hopping, the difference in negative COM displacement $(3.9 \% ; P=0.002)$ was only marginally greater than the difference in vGRF. During bilateral hopping it would therefore appear that $\mathrm{K}_{\mathrm{vert}}$ asymmetries are a consequence of asymmetries in both VGRF and negative COM displacement that are of a similar magnitude. During the bilateral and unilateral drop jump tasks, between-limb differences in negative COM displacement were substantially larger 
than differences in vGRF $(25.1 \%$ and $19.8 \%$, respectively; both $P=0.002)$; Kvert asymmetries during these maximal drop jump tasks appears to be a greater consequence of differences in negative COM displacement. The negative COM displacement observed during bilateral hopping in the current study $(\sim 0.10 \mathrm{~m})$ is comparable to figures reported in other investigations (Hobara et al., 2014; Joseph et al., 2013) but was substantially greater than figures observed during drop jumping. As it is possible that the lack of a controlled hopping frequency contributed to this effect, Joseph et al. (2013) have previously reported that displacement may be increased when participants hop at a self-selected frequency and may partially explain why the discrepancies observed in the current study were so large. It is recommended that future studies familiarise participants with this task until they are able to satisfactorily perform hopping tasks at a set frequency. Nonetheless, it is not unexpected that the higher vGRFs associated with drop jumping may result in less negative COM displacement. For example, it has been reported that displacement during bilateral hopping is reduced as hopping frequency, and subsequent vGRF, are increased (Hobara et al., 2013; Hobara et al., 2014).

In conclusion, all three types of performance task demonstrate the potential to detect $\mathrm{K}_{\text {vert }}$ asymmetry; such asymmetries may be greatest in bilateral drop jumping and lowest in bilateral hopping. Kvert asymmetry has been linked to an increased incidence of soft-tissue injury (Pruyn et al., 2012) and has been hypothesised to impair athletic performance as the application of force to each limb may be imbalanced (Wilson et al., 1994). Although further research is required to fully explore the impact of Kvert asymmetry on injury incidence and athletic performance, it would appear prudent to screen individuals for Kvert asymmetry as this is a highly trainable and modifiable parameter. It is recommended that practitioners and 
researchers use the performance task that most closely matches the demands of an individual's sport. 


\section{References}

Arampatzis, A., Schade, F., Walsh, M., \& Brüggemann, G. P. (2001). Influence of leg stiffness and its effect on myodynamic jumping performance. Journal of Electromyography and Kinesiology, 11(5), 355-364.

Bachman, G., Heise, G. D., \& Bressel, E. (1999). The symmetry of the human leg spring: spring coefficients between right and left legs during running. Paper presented at the Proceedings of the 23rd Annual Meeting of the American Society of Biomechanics, Pittsburgh, Philadelphia.

Benjanuvatra, N., Lay, B. S., Alderson, J. A., \& Blanksby, B. A. (2013). Comparison of ground reaction force asymmetry in one- and two-legged countermovement jumps. Journal of Strength and Conditioning Research, 27(10), 2700-2707.

Blazevich, A. J. (2007). Position, Velocity and Acceleration. In A. J. Blazevich (Ed.), Sports Biomechanics: The Basics: Optimising Human Performance (pp. 1-14). London, England: A \& C Black Publishers Ltd.

Brughelli, M., \& Cronin, J. (2008). Influence of running velocity on vertical, leg and joint stiffness: modelling and recommendations for future research. Sports Medicine, 38(8), 647-667.

Butler, R. J., Crowell III, H. P., \& Davis, I. M. (2003). Lower extremity stiffness: implications for performance and injury. Clinical Biomechanics, 18(6), 511517.

Cohen, J. (1998). Statistical power analysis for the behavioral sciences (2nd ed.). New Jersey: Lawrence Erlbaum.

Coleman, D. R., Cannavan, D., Horne, S., \& Blazevich, A. J. (2012). Leg stiffness in human running: Comparison of estimates derived from previously published models to direct kinematic-kinetic measures. Journal of Biomechanics, 45(11), 1987-1991.

Divert, C., Baur, H., Mornieux, G., Mayer, F., \& Belli, A. (2005). Stiffness adaptations in shod running. Journal of Applied Biomechanics, 21(4), 311-321.

Farley, C. T., Glasheen, J., \& McMahon, T. A. (1993). Running springs: speed and animal size. The Journal of Experimental Biology, 185, 71-86.

Farley, C. T., \& Morgenroth, D. C. (1999). Leg stiffness primarily depends on ankle stiffness during human hopping. Journal of Biomechanics, 32(3), 267-273.

Flanagan, E. P., \& Comyns, T. M. (2008). The use of contact time and the reactive strength index to optimize fast stretch-shortening cycle training. Strength \& Conditioning Journal, 30(5), 32-38.

Flanagan, E. P., \& Harrison, A. J. (2007). Muscle dynamics differences between legs in healthy adults. Journal of Strength and Conditioning Research, 21(1), 6772.

Heise, G. D., \& Bachman, G. (2000). Leg spring model properties of children. Paper presented at the Proceedings of the 24th Annual Meeting of the American Society of Biomechanics, Pittsburgh, Philadelphia.

Hobara, H., Inoue, K., \& Kanosue, K. (2013). Effect of hopping frequency on bilateral differences in leg stiffness. Journal of Applied Biomechanics, 29(1), 55-60.

Hobara, H., Inoue, K., Kobayashi, Y., \& Ogata, T. (2014). A comparison of computation methods for leg stiffness during hopping. Journal of Applied Biomechanics, 30(1), 154-159. 
Hobara, H., Kimura, K., Omuro, K., Gomi, K., Muraoka, T., Iso, S., \& Kanosue, K. (2008). Determinants of difference in leg stiffness between endurance- and power-trained athletes. Journal of Biomechanics, 41(3), 506-514.

Hobara, H., Kimura, K., Omuro, K., Gomi, K., Muraoka, T., Sakamoto, M., \& Kanosue, K. (2010). Differences in lower extremity stiffness between endurance-trained athletes and untrained subjects. Journal of Science and Medicine in Sport, 13(1), 106-111.

Joseph, C. W., Bradshaw, E. J., Kemp, J., \& Clark, R. A. (2013). The interday reliability of ankle, knee, leg, and vertical musculoskeletal stiffness during hopping and overground running. Journal of Applied Biomechanics, 29(4), 386-394.

Lloyd, R. S., Oliver, J. L., Hughes, M. G., \& Williams, C. A. (2009). Reliability and validity of field-based measures of leg stiffness and reactive strength index in youths. Journal of Sports Sciences, 27(14), 1565-1573.

Marshall, B. M., \& Moran, K. A. (2013). Which drop jump technique is most effective at enhancing countermovement jump ability, "countermovement" drop jump or "bounce" drop jump? Journal of Sports Sciences, 31(12), 1368-1374.

Moresi, M. P., Bradshaw, E. J., Greene, D. A., \& Naughton, G. A. (2015). The impact of data reduction on the intra-trial reliability of a typical measure of lower limb musculoskeletal stiffness. Journal of Sports Sciences, 33(2), 180-191.

Padua, D. A., Arnold, B. L., Carcia, C. R., \& Granata, K. P. (2005). Gender differences in leg stiffness and stiffness recruitment strategy during twolegged hopping. Journal of Motor Behaviour, 37(2), 111-126.

Pearson, S. J., \& McMahon, J. (2012). Lower limb mechanical properties: determining factors and implications for performance. Sports Medicine, 42(11), 929-940.

Pruyn, E. C., Watsford, M., \& Murphy, A. (2014). The relationship between lowerbody stiffness and dynamic performance. Applied Physiology, Nutrition, and Metabolism, 39(10), 1144-1150.

Pruyn, E. C., Watsford, M. L., Murphy, A. J., Pine, M. J., Spurrs, R. W., Cameron, M. L., \& Johnston, R. J. (2012). Relationship between leg stiffness and lower body injuries in professional Australian football. Journal of Sports Sciences, $30(1), 71-78$.

Rhea, M. R. (2004). Determining the magnitude of treatment effects in strength training research through the use of effect size. Journal of Strength and Conditioning Research, 18(4), 918-920.

Robinson, R. O., Herzog, W., \& Nigg, B. M. (1987). Use of force platform variables to quantify the effects of chiropractic manipulation on gait symmetry. Journal of Manipulative Physiological Therapy, 10(4), 172-176.

Simon, A. M., \& Ferris, D. P. (2008). Lower limb force production and bilateral force asymmetries are based on sense of effort. Experimental Brain Research, 187(1), 129-138.

Wilson, G. J., Murphy, A. J., \& Pryor, J. F. (1994). Musculotendinous stiffness: Its relationship to eccentric, isometric, and concentric performance. Journal of Applied Physiology, 76(6), 2714-2719.

Zifchock, R. A., Davis, I., Higginson, J., \& Royer, T. (2008). The symmetry angle: A novel, robust method of quantifying asymmetry. Gait \& Posture, 27(4), 622627. 
